\title{
A fish stock assessment model using survey data when estimates of catch are unreliable
}

\author{
R.M. Cook* \\ MASTS Marine Population Modelling Group, Department of Mathematics and Statistics, University of Strathclyde, Livingstone Tower, 26 Richmond Street, Glasgow, G1 1 XH, \\ Scotland, UK
}

\section{A R T I C L E I N F O}

\section{Article history:}

Received 18 June 2012

Received in revised form

14 December 2012

Accepted 13 January 2013

\section{Keywords:}

Bayesian model

Stock assessment

Misreported catch

Natural mortality

Haddock

Survey

\begin{abstract}
A B S T R A C T
Methods of assessment that depend upon commercial catch data can be undermined by misreporting or where parts of the catch, such as discards, are not accounted for. An age-structured model that makes use of survey data alone, and avoids this problem, is developed within a Bayesian framework so that routine stock summary statistics such as fishing mortality, recruitment and spawning stock biomass can be estimated with associated levels of uncertainty. It is also possible to estimate catch on a relative scale which can be compared to reported catches. The model is applied to West of Scotland haddock (Melanogrammus aeglefinus), a stock with suspected high catch misreporting. Stock trends derived from the model are consistent with conventional assessments that use catch data during periods of low misreporting. Estimated proportions of fish at each age in the catch correspond closely with observed values. Model estimates of total catches suggest substantial misreporting in some years, though the precision of the estimates is very low. Revised estimates of natural mortality are obtained from the model that are higher than conventional values used for this stock. These new values are generally consistent with those obtained from multispecies predation modelling for the adjacent North Sea stock. The model provides many of the basic quantities used for management advice. It should not be regarded as a replacement for more comprehensive analyses, but an additional tool to explore available data when catch information is unreliable.
\end{abstract}

(C) 2013 Elsevier B.V. All rights reserved.

\section{Introduction}

The aspiration of many stock assessments is to be able to estimate, on an absolute scale, the number of fish at each age in the stock and the associated fishing mortality. From these quantities it is possible to calculate spawning stock biomass and recruitment, and possibly infer a stock-recruitment relationship from which the full dynamics of the stock can be modelled. Such assessments frequently use age composition data from the landed catch which forms the core of the analysis. Some early assessment methods relied entirely on catch at age data, for example, virtual population analysis (VPA) (Gulland, 1965; Pope and Shepherd, 1982) and require ad hoc assumptions about initial values to perform the calculations. It is recognised that auxiliary information in the form of indices of abundance, greatly improve assessments by avoiding the need to make arbitrary assumptions about initial values (Doubleday, 1981; Deriso et al., 1985; Gavaris, 1988; Methot, 1990; Shepherd, 1999). These methods quickly became standard approaches to assessment and now form the everyday tools of

\footnotetext{
* Tel.: +44 141548 3666; fax: +441415483345.

E-mail address: robin.cook@strath.ac.uk
}

fishery scientists. In many respects it is the auxiliary information fishery independent data - which is essential and may be sufficient to perform an adequate assessment even when catch data are absent.

The ubiquitous use of catch at age data arises partly because it is often relatively inexpensive to collect and sampling can be done more easily on land, but also because it is the main source of information that scales any abundance estimate to an absolute value. However, not all of the catch is landed (FAO, 1994) so often only part of the catch is sampled. Furthermore, where catch controls are used to manage a fishery, the recorded catch is often distorted by actions to circumvent the regulations. As a result, not only may the scaling be distorted but variable bias in recorded catches may undermine the veracity of any assessment. In the European Union jurisdiction, many assessments have been abandoned because recorded catches are regarded as unreliable (ICES, 2010) leaving managers with little evidence upon which decisions can be based. Methods of assessment that can provide evidence of stock status to managers that are not dependent on catch data are therefore required.

Where abundance indices are available, for example from research vessel surveys, a possible solution to this difficulty is to assess fishing mortality and stock trends on a relative scale (Cook, 1997) without the use of catch data. Assessments using survey data 
only may not perform as well as those that include catch at age data (Patterson, 1998; Hammond and Trenkel, 2005) but where the catch data are compromised, these approaches may offer a useful alternative. In many assessments carried out by the International Council for the Exploration of the Sea (ICES) a survey-only method, SURBA, (Needle, 2002; Beare et al., 2005) is used. One particular difficulty of these models is that selectivity, natural mortality, and survey catchability parameters may be confounded and a conventional least squares fit of the model requires assumptions about one or more of these in order to obtain a unique solution. In practice, there is often some information on all of these quantities, although it may not be very precise, which could be used to aid fitting the model and hence estimate fishing mortality and relative catch. Using a Bayesian approach where informative prior information can be utilised offers a means of developing these models both to estimate quantities of interest and also to obtain appropriate estimates of uncertainty. In this paper a model is developed for the analysis of one or more surveys using prior information on fleet selectivity and natural mortality to estimate stock trends, relative catch and fishing mortality. The model is applied to a stock, haddock (Melanogrammus aeglefinus) in ICES Division VIa, where misreported catch is believed to be substantial and compared to the ICES assessment which is the recognised international consensus on the status of the stock. The ICES assessment attracts criticism because of the perceived unreliability of the reported catches. Results from the model developed here, which does not use these data, suggest the estimates of the quantities of interest could form the basis for fishery management advice and hence avoid the problems associated with biased catch data (ICES, 2010).

\section{Assessment model}

We assume at least one survey is available that provides an age structured index of abundance. In principle, even if the index is on a relative scale, measures of cohort abundance in successive time intervals will provide information on the total mortality experienced by the population. The task is to parameterise the mortality and the cohort signal in a way which accounts for sampling efficiency and measurement errors in the data so that the underlying abundance of the stock and its associated mortality rates can be estimated.

\subsection{Structural model}

The population, $N$, is assumed to decay with a total mortality $Z$ according to the conventional exponential equation:

$N_{a+1, y+1}=N_{a, y} \mathrm{e}^{-z_{a, y}}$

where $a$ is an index for age and $y$ is an index for year.

The total mortality is partitioned between fishing mortality $F$ and natural mortality $M$ so that:

$Z_{a, y}=F_{a, y}+M_{a, y}$

The total catch in number, $C$, of all fish taken by the fishery is assumed to follow the Baranov catch equation:

$C_{a, y}=\frac{F_{a, y} N_{a, y}\left(1-\mathrm{e}^{-Z_{a, y}}\right)}{Z_{a, y}}$

In common with many fishery models we assume that the fishing mortality can be expressed as the product of an age effect or selectivity, $s$, and a year effect, $f$. Selectivity is the proportion of fish at each age entering the gear that are retained, while the year effect is a measure of the overall fishing mortality at fully selected age groups (i.e. when $s=1$ ). Denoting commercial fishery selectivity by $s^{*}$ the annual fishing mortality at age is given by:

$F_{a, y}=s_{a, y}^{*} f_{y}$

The change in fishing mortality rate from year to year is likely to be fishery specific and will depend on how it is managed. In general it might be expected that inertia in commercial fleets will mean that annual changes in $F$ will be limited. It is assumed here that the year effect follows a simple time series model with a multiplicative random effect, $\varepsilon$ :

$f_{y}=f_{y-1} \mathrm{e}^{\varepsilon y}$

and

$\varepsilon_{y} \sim \operatorname{Normal}\left(0, \sigma^{*}\right), \quad y \neq 1$

where $\sigma^{*}$ is the standard deviation of an irregular fluctuation associated with the commercial fleet. In effect it assumes that the time series of $\log$ differences in $f$ is a stationary white noise process. Large values $\sigma^{*}$ mean that the fishing mortality can exhibit large annual fluctuations.

While abundance indices may contain information on the annual change in mortality, they will not contain sufficient information to estimate selectivity at age freely because there is no data related directly to the catch. Hence, it is probably better to model selectivity with a relatively stiff function with few parameters for which informative priors can be specified. For trawl fisheries a common assumption, adopted here, is to use a standard two-parameter logistic selection curve where the proportion retained in the gear is a function of length. For ease of interpretation the selectivity function is parameterised in the form of the $50 \%$ retention length, $L_{50}$ and selection range, $s r$. Hence for the commercial fishery, selectivity is taken to be dependent on mean length at age, $\bar{l}$ :

$\operatorname{logit}\left(s_{a, y}^{*}\right)=\left(\frac{\ln (9)}{s r^{*}}\right) \bar{l}_{a, y}-\frac{L_{50}(\ln (9)}{s r^{*}}$

The other component of total mortality is natural mortality, $M$. It is unusual to find data that can be incorporated into a stock assessment in order to estimate natural mortality within the model. Lee et al. (2011) argue, based on simulation studies, that $M$ can be estimated from stock assessment data provided the model is specified correctly. Where only survey data are used, it is unlikely that it is possible to estimate $M$ since there are no observations on catch to partition total mortality. It is preferable to assume some knowledge of $M$ in order to be able to estimate $F$. Natural mortality has been the subject of a number of reviews (Pauly, 1980; Vetter, 1988; Lorenzen, 1996, 2000) and empirical methods have been suggested for its estimation (Hoenig, 1983; Myers and Doyle, 1983). In the model described here, results of a meta-analysis of worldwide fish stocks by Lorenzen (1996) are used for their simplicity where natural mortality is related to weight. In particular it is assumed that $M$ is a function of mean weight at age, $\bar{w}$ :

$M_{a, y}=\alpha\left(\bar{w}_{a, y}\right)^{\beta}$

where $\alpha$ and $\beta$ are constants that mediate the change of $M$ with age.

\subsection{Observation equations}

The indices of abundance, $U$, from surveys will be related to the true population in some way. This relationship may not be linear (Gudmundsson, 2004). However, a non-linear relationship increases the number of parameters and the degrees of freedom, and with noisy data are unlikely to be adequately estimated. For parsimony, it is assumed $U$ is directly proportional to population size, where the proportionality constant is the product of an age 
specific selectivity, $s$, and an overall survey catchability $q$. If $\lambda$ is a discount factor to account for mortality during the year up to the sampling time of the survey, then the index of abundance for the $k$ th survey can be expressed as:

$U_{a, y, k}=s_{a, k} q_{k} N \lambda_{a, y, k}$

Specifically, if $\rho$ is the proportion of the year elapsed before the survey:

$\lambda_{a, y, k}=\mathrm{e}^{-\rho_{k} Z_{a, y}}$

Survey selectivity presents a similar problem of estimation as commercial fleet selectivity. Where the abundance index is derived from trawl sampling, the simplest model is to assume a logistic curve. In the example stock considered here, data on length are not available for the surveys, so age is used as a proxy for size. Using the $50 \%$ selection age as $A_{50}$, and selection range by $s r$, selectivity is given by:

$\operatorname{logit}\left(s_{a, k}\right)=\left(\frac{\ln (9)}{s r_{k}}\right) a-\frac{A_{50, k} \ln (9)}{s r_{k}}$

The survey indices will be observed with an associated error. Clearly the sample distribution must be non-negative and it is usually regarded as right-skewed. For simplicity it is assumed that $U$ is observed from a lognormal distribution. Writing $u=\log (U)$, the observed values $\hat{u}$ are drawn from a normal distribution with mean $\mu$ and standard deviation $\sigma$ :

$\hat{u}_{a, y, k} \sim \operatorname{Normal}\left(u_{a, y, k}, \sigma_{a, y, k}\right)$

\subsection{Prior distributions}

It is necessary to specify priors for the parameters in the model. These are the initial year effect, $f$, the random effect on fishing mortality, initial populations in year 1 and fish of the youngest age in all years, selectivity parameters, survey catchability, the natural mortality relationship and the standard deviation of the abundance index sample distribution. The choice of prior will be dependent on the stock and fishery concerned. In this paper West of Scotland haddock is used to exemplify application of the model and priors are proposed with that in mind.

Experience of similar fisheries in the region suggests that overall fishing mortality in year $y=1$ (1985) is likely to be in the range 0.3-1.5 (ICES, 2011a). Since fishing mortality is greater than zero, a uniform prior is used to include this interval:

$\ln \left(f_{1}\right) \sim$ Uniform $(-3,1)$

The irregular fluctuation in annual fishing mortality, $\sigma^{*}$, was set to 0.22 which means that most of the annual change in fishing mortality will be limited to the $95 \%$ interval $(0.65 f, 1.55 f)$.

The prior distribution for $L_{50}$ was assumed to be approximately normal with the mean given by the legal minimum landing size (mls) which for this stock is $30 \mathrm{~cm}$. It might be expected the fleets would set their $50 \%$ retention near the mls to avoid catching fish that would have to be discarded, yet retain a high proportion of marketable fish. The variance was set large to reflect relative lack of knowledge about the true $L_{50}$. A variance of 60 was used which gives a $95 \%$ range of approximately $10-45 \mathrm{~cm}$ :

$L_{50} \sim \operatorname{Normal}(\mathrm{mls}, \sqrt{60})$

Selection range was also assumed to be normal. Numerous studies have been performed on gear selectivity of haddock. These are usually experiments conducted to test individual gears rather than fleets. Estimates of selection range are highly variable (Graham et al., 2004; Özbilgin et al., 2006). A typical value of $6 \mathrm{~cm}$ was chosen for the mean by inspecting values from these selectivity experiments and due to the high variability a large standard deviation was used:

$s r^{*} \sim \operatorname{Normal}(6, \sqrt{2})$

The parameters in the natural mortality-weight function were taken from the "ocean fish" in Lorenzen (1996) which gives means and $95 \%$ confidence intervals of the estimates. The confidence intervals were used to approximate a normal distribution for each parameter:

$\alpha \sim \operatorname{Normal}(3.69, \sqrt{0.25})$

$\beta \sim \operatorname{Normal}(-0.305, \sqrt{0.0008})$

There is little prior information on the size of the initial populations since recruitment is highly variable and unpredictable. The survey indices should be rich in information about the size of these populations so the choice of prior is unlikely to influence the posterior distributions. Consequently the log of the initial populations in year $y=1$ and at age $a=1$ in all years were drawn from a uniform distribution:

$\ln \left(N_{1, y}\right) \sim$ Uniform $(-2,11)$

$\ln \left(N_{a, 1}\right) \sim$ Uniform $(-2,11)$

For the surveys selectivity is assumed to be by age. For $A_{50}$, if all fish are fully selected at the youngest age, negative values are possible. In the absence of any information on age selectivity, it was drawn from a uniform prior in the age range:

$A_{50, k} \sim$ Uniform $(-3,6)$

Similarly, the survey selection range is assumed to be drawn from a uniform distribution:

$s r_{a, k} \sim$ Uniform $(0.1,6)$

Conventional non-informative priors were set for the inverse survey variances using a gamma distribution:

$\frac{1}{\sigma_{a . k}} \sim \operatorname{Gamma}(0.1,0.1)$

Finally, survey catchability was drawn from a uniform prior. In practice at least one survey catchability value has to be fixed in order to estimate the others. It was assumed that the log catchability of the Scottish quarter 1 (i.e. $k=1$ ) survey was zero. For the remaining surveys:

$\ln \left(q_{k}\right) \sim$ Uniform $(-7,5), \quad k \neq 1$

\section{Materials and methods}

\subsection{Data}

In the West of Scotland haddock fish typically mature by the age of three when they reach a length of about $35 \mathrm{~cm}$. The maximum age is around 20 years but at current levels of exploitation few fish survive beyond 7 years (ICES, 2011a). They are caught predominantly in bottom trawls in a mixed fishery for roundfish.

Survey data were taken from the most recent ICES assessment report (ICES, 2011a). The four surveys concerned and the year and age range used are given in Table 1 . These are the same ranges as was used in the ICES assessment except for the inclusion of 0-group indices. An arbitrary value of 0.01 was added to the index to overcome zero values when taking logs. However, nearly all the index values were greater than 1 . A survey value for 2011 for the Sco1 
Table 1

Trawl surveys in ICES Division VIa used in the analysis. Values of the indices are given in ICES (2011a).

\begin{tabular}{llll}
\hline Survey & Abbreviation & $\begin{array}{l}\text { Year range } \\
\text { used }\end{array}$ & $\begin{array}{l}\text { Age range } \\
\text { used }\end{array}$ \\
\hline Scottish quarter 1 survey & Sco1 & $1985-2010$ & $1-7$ \\
Scottish quarter 4 survey & Sco2 & $1996-2009$ & $0-7$ \\
Irish quarter 4 survey & Ire1 & $1993-2002$ & $0-7$ \\
Irish quarter 4 survey, revised & Ire2 & $2003-2010$ & $0-7$ \\
\hline
\end{tabular}

survey is available but changes to the survey design and sampling gear in that year render it inconsistent with the previous survey series and was excluded.

Data on mean weights and proportion mature at age were taken from the same source. The mean weights used were the stock weights which were applied both for the calculation of spawning stock biomass and total catch in weight (yield). Estimates of observed proportion at age in the total catch were derived from the total catch at age in ICES (2011a).

In order to estimate the selectivity of the commercial fleet, mean length-at-age is required. There is no easily accessible source of mean lengths for international catch data. Consequently, mean length at age was derived from mean weight at age using the inverse weight-length relationships reported by Coull et al. (1989). This will give biased estimates of the true mean length at age, but it should give an adequate index of size for the purpose of estimating total fleet selectivity.

\subsection{Model fitting and summary statistics}

WinBUGS 1.4 (Lunn et al., 2000) was used to fit the model. It automatically sets a minimum burn in period of 4000 iterations. Exploratory runs with 3 sampling chains and between 10,000 and 20,000 iterations indicated that the chains converged very rapidly and that the means of the parameters changed little after about 5000 iterations. Posterior distributions were therefore calculated from a single chain of 10,000 iterations with a burn in period of 4000 iterations. The model code is given in the Appendix.

Standard stock summary statistics were calculated within the fitting procedure to obtain median values and 95\% credible intervals. The principal quantities concerned are mean annual fishing mortality, $\bar{F}$, spawning stock biomass (SSB) and total catch in weight, $Y . \bar{F}$ is defined as:

$\bar{F}=\frac{1}{a 2-a 1+1} \sum_{a 1}^{a 2} F_{a}$

where $a 1$ and $a 2$ represent the reference age range for the mean, usually on the fully selected ages. For this stock the mean over ages 2-6 was used as this is the conventional range used by ICES for this particular stock. Spawning stock biomass, where $p_{a}$ is the proportion of fish mature at age $a$, is given by:

$\mathrm{SSB}=\sum_{a} p_{a} \hat{w}_{a} N_{a}$

and total catch in weight is:

$Y=\sum_{a} \hat{w}_{a} C_{a}$

\subsection{Comparisons with ICES assessment}

Output from the analysis was compared to the most recent available ICES assessment (ICES, 2011a) which is the internationally agreed consensus. The ICES assessment model uses catch at age data and omits the two Irish survey series used here. It makes different model structural assumptions. A Beverton-Holt relationship is used to constrain recruitment, and natural mortality is assumed to be a known constant (0.2). The ICES model assumes that selectivities change over time but independently of fish size. In the model presented here selectivity is size dependent not time dependent. In comparing the current model to the ICES assessment we are therefore concerned to see whether a greatly reduced data set, and the necessary assumptions that are associated with fewer data, can still produce credible estimates of the management values of interest.

For fishing mortality the measurement scale of the current model and the ICES method (Fryer, 2001) will be the same, but scales for recruitment and biomass values such as SSB and catch will differ as the ICES assessment estimates absolute quantities conditioned on assumptions about natural mortality and catch. In order to compare trends, the ICES values for recruitment at age 1, catch (in weight) and biomass were re-scaled to the Sco1 (see Table 1) reference survey using the ratio of the time series means for the reference period 1985-1994 when misreporting was regarded by ICES as low or negligible (ICES, 2011a).

Estimates of misreported catch (or "missing" catch) were obtained by scaling up the total catch estimates from the model to absolute values using the inverse ratio of the means as described above and then subtracting the observed total catch from these. Comparable estimates from the ICES assessment were obtained by subtracting the observed total catch from the catch estimated from the ICES assessment model. Model estimates of total and missing catch were then compared with reported catches and estimates of misreporting from the ICES assessment.

Provided the assumption in the model relating to commercial fleet selectivity is adequate, it is possible to obtain estimates of the total catch and catch at age. The reported total catches are believed to suffer from substantial misreporting in some years which means numbers at age in the catch will be biased for these years because catch is used as a factor to raise stratified samples of landed catch age compositions to obtain total numbers at age. However, the proportions at age in the catch should not be affected by misreporting bias since there is no application of a (biased) catch raising factor to the samples. Estimated proportions at age in the catch were therefore compared to the recorded proportions at age as an external check on model performance. Because observed numbers at age 0 are derived almost entirely from estimates of discards which are known to be very imprecise (Stratoudakis et al., 1999), only ages 1-7 were considered here.

In order to compare selectivity estimated from the current model with the ICES estimates it is necessary to reconcile the size dependent selectivity assumed here with the age dependent selectivity assumed in the ICES model. In order to do this, posterior selectivities by age, based on the mean length at age for the reference period 1985-1994, were compared with the ICES estimates of mean fishing mortality at age over the same reference years scaled to the age 7 mean $F$ to give an estimate of relative selectivity.

The availability of a number of surveys for analysis is fortunate for any assessment and it raises the question of the value of multiple data sets. To examine this, the analysis described here included two runs of the model, one with only the Scottish quarter 1 survey and a full analysis with all survey data available. The ratio of the coefficient of variation (cv) for the multiple survey run to the single survey was calculated as a measure of the change in precision to compare the two runs. Sco1 was used as the reference survey since it is the longest running time series.

Conventionally, natural mortality for this stock has been assumed to be 0.2 for all ages. For the adjacent stock in the North Sea estimates of natural mortality have been made using multispecies virtual population analysis (MSVPA) (Andersen and Ursin, 1977; Pope, 1979). These estimates are based on stomach sampling 


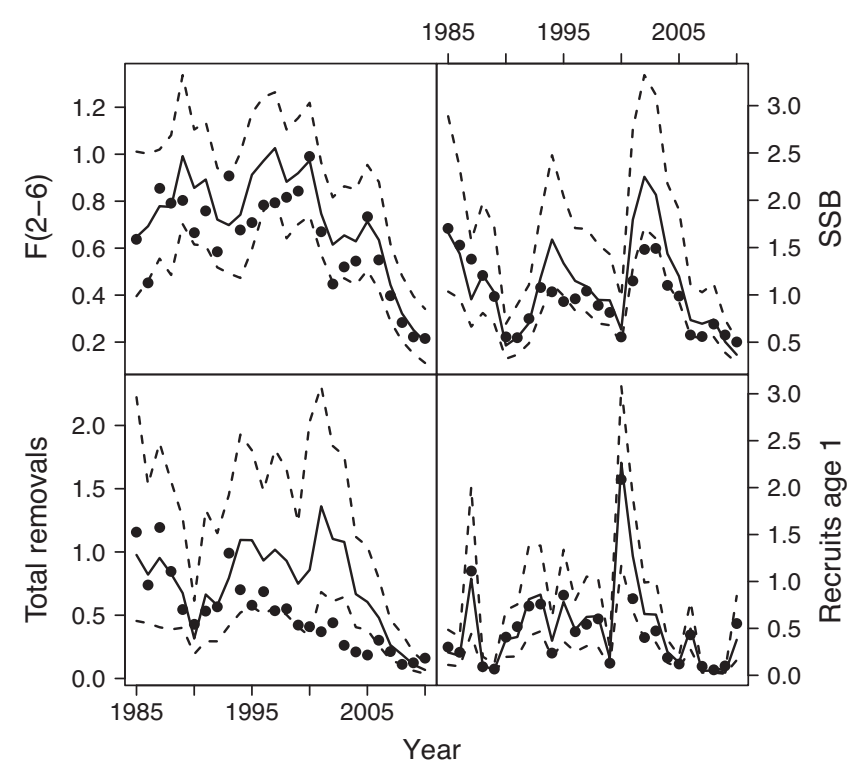

Fig. 1. Trends estimated from the model (heavy line) and the ICES assessment (dots) for mean fishing mortality (ages 2-6), spawning stock biomass, recruitment at age 1 and estimated total catch. Dashed lines show the 95\% credible interval. Apart from $F(2-6)$ the ICES values are rescaled to the Sco 1 survey and are in arbitrary cpue units. In the case of total removals, the dots indicate total reported catch.

programmes that provide data to estimate mortality due to predation and are generally regarded as the best estimates of $M$ for North Sea haddock. To check that the $M$ values obtained from the current model are realistic they have been compared to MSVPA North Sea values. Values of $M$ based on mean weights at age for the 1985-1994 reference period were used to compare with the equivalent values of $M$ as used for the North Sea based on MSVPA (ICES, 2011b).

\section{Results}

\subsection{Stock summary}

The estimates of fishing mortality are very similar in their magnitude and trends to the ICES assessment (Fig. 1) with $F(2-6)$ showing a marked decline in recent years. The ICES values lie well within the $95 \% \mathrm{CI}$ estimated from the model indicating consistency between the analyses. For SSB there is close agreement between the two assessments in the early period but with the ICES values somewhat lower in the last decade. This may reflect the choice of scaling period (1985-1994) or possibly the effect of using higher natural mortality values in the current model. Recruitment trends are almost identical in the two assessments with the ICES values on or within the $95 \% \mathrm{CI}$ estimated from the model.

The trends in reported catch are almost identical during both the early and late periods when misreporting is believed to have been low suggesting these are adequately estimated by the model (Fig. 1). However, between 1995 and 2005 the observed catch series falls well below the estimated trend and for much of this period is outside the $95 \% \mathrm{CI}$ estimated from the model. This provides some evidence to support the view that reported values are affected by "missing" catch, probably as the result of misreporting.

\subsection{Survey indices}

The log survey indices appear to be fitted reasonably well in all four surveys, helped by the very strong recruitment signal in the data (Fig. 2). Estimated measurement error for each survey is lowest for the intermediate age groups and tends to be lower for

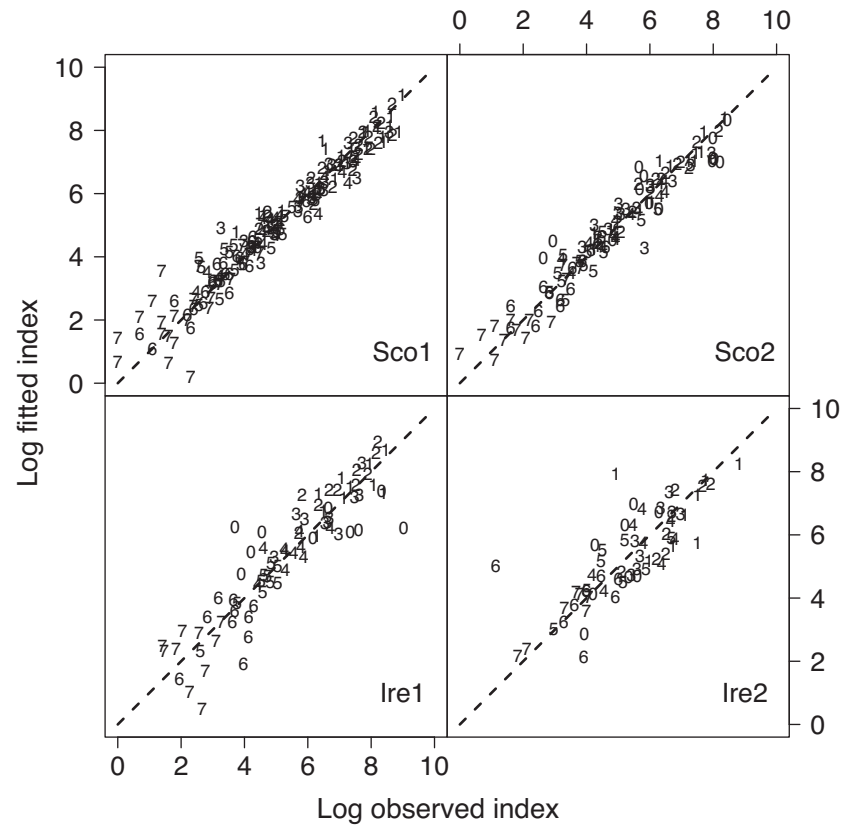

Fig. 2. The fitted log index plotted against the observed values for the four research vessel surveys. Numbers on the plot indicate age classes. The dashed line shows the line $y=x$.

the two Scottish surveys (Table 2). The lower errors associated with intermediate ages reflects the larger numbers of fish sampled at these ages and their lower exposure to changes in selectivity due to variations in growth.

The survey data appear to contain information on relative catchability since the uniform priors have been updated to give clearly defined modes as can be seen in Fig. 3 which shows the posterior distributions for the three surveys relative to Sco1. With the exception of the Ire1, all surveys appear to have similar catchability since $\log q=0$ is contained in the posterior distribution. The Ire 1 survey has both a higher catchability and a broader posterior distribution.

\subsection{Selectivities}

There appears to be some information in the data to estimate survey selectivity. The selectivity parameter priors are uniform while the posteriors show distinct modes substantially updating the priors (Fig. 4). The age dependent selectivities of the surveys (Fig. 5) show that fish are largely selected by age 3 in the Sco1 and Ire2 surveys. Selectivity on the youngest age for these surveys is not precisely estimated and there is only weak evidence that selectivity is below 1 for Sco1. For Sco2 and Ire1, selectivity is greater (i.e. fewer young fish are selected) and fish are only fully selected at the oldest ages. This may be an effect of the spatial distribution of the fish in the later part of the year differing from the spring distribution, rather than a size/gear effect.

Table 2

Estimates of the measurement error (variance) associated with each survey series.

\begin{tabular}{lllll}
\hline Age & Sco1 & Sco2 & Ire1 & Ire2 \\
\hline 0 & & 0.917 & 2.675 & 1.436 \\
1 & 0.384 & 0.143 & 0.403 & 2.044 \\
2 & 0.250 & 0.108 & 0.495 & 0.455 \\
3 & 0.362 & 0.387 & 0.370 & 0.324 \\
4 & 0.199 & 0.133 & 0.260 & 0.693 \\
5 & 0.737 & 0.250 & 0.130 & 0.582 \\
6 & 0.289 & 0.221 & 0.917 & 2.574 \\
7 & 0.882 & 0.342 & 1.191 & 0.195 \\
\hline
\end{tabular}




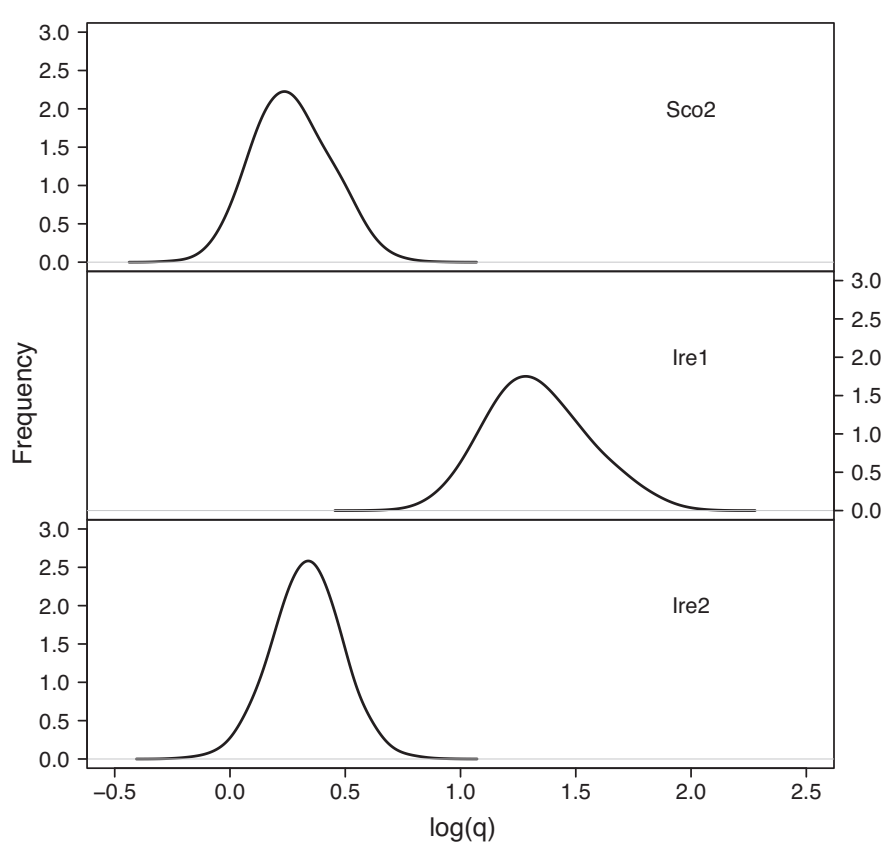

Fig. 3. Posterior distributions of $\log (q)$, the survey catchability relative to Sco1. The prior distribution is uniform for all three surveys on the interval (-7.5).
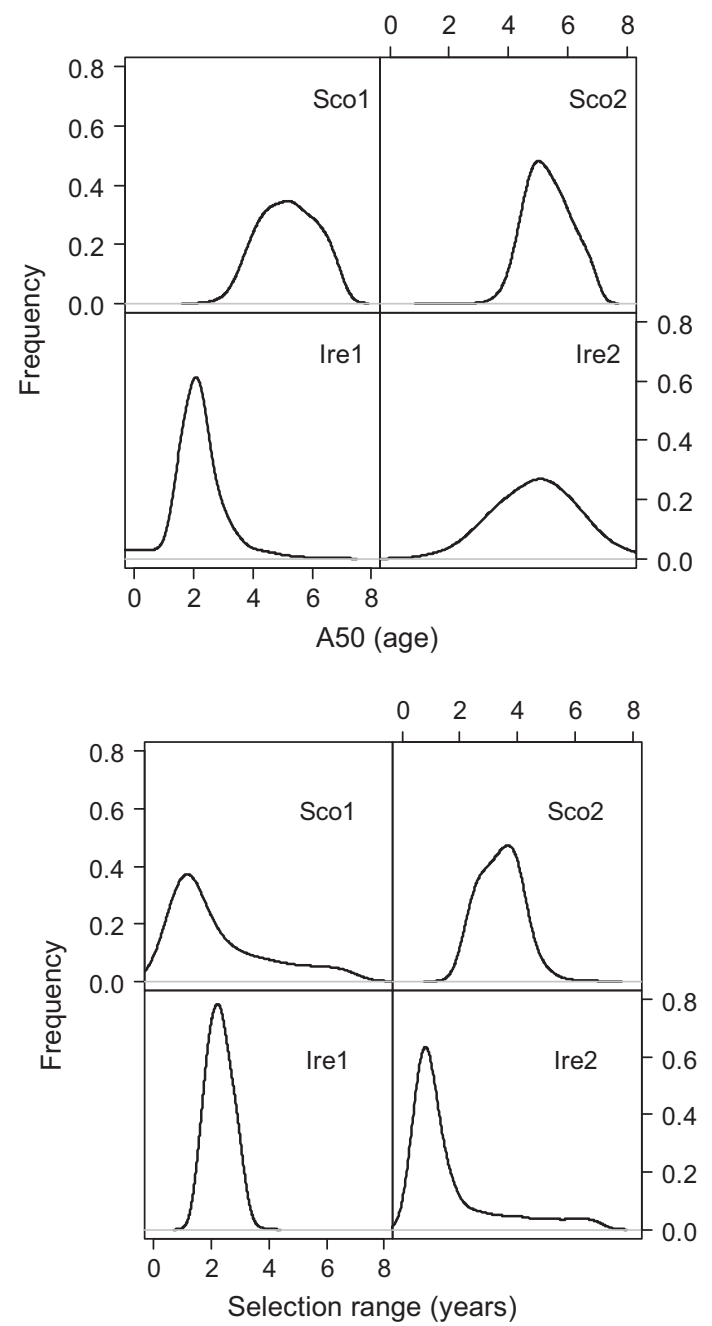

Fig. 4. The posterior distributions for $A_{50}$ (upper panel) and $s r$ (lower panel), the selectivity parameters for the surveys. In all four surveys the prior distributions are uniform.

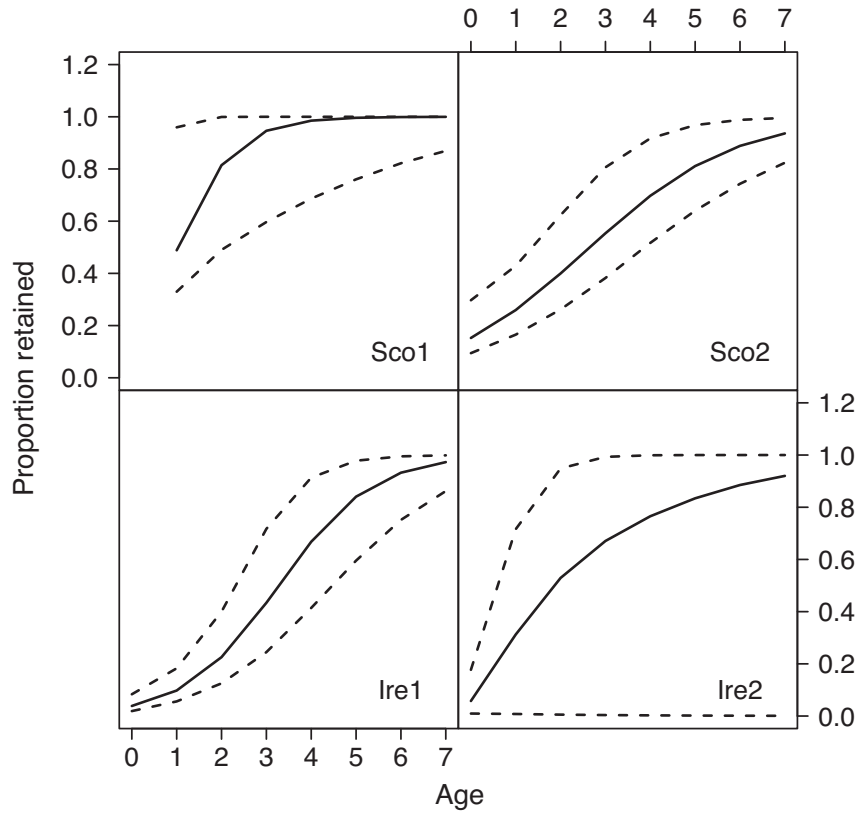

Fig. 5. Estimated selectivity for the four surveys. The median value is shown as a solid line and the dashed lines show the $95 \%$ credible interval.

The model estimates of fleet selectivity parameters show some change from the assumed prior means. For $L_{50}$, the mean has declined and its standard deviation is much smaller but the selection range posterior distribution differs little from the prior (Fig. 6). Although the posterior mean $L_{50}$ is only modestly different from the prior, it appears to be a better descriptor of selectivity as can be seen from Fig. 7. This shows the prior and posterior selectivities by age compared with the ICES assessment (which uses commercial catch data). Clearly the posterior estimates for fish aged 2 and older from the model agree well with estimates using catch data, but there is still a large difference at age 1 .
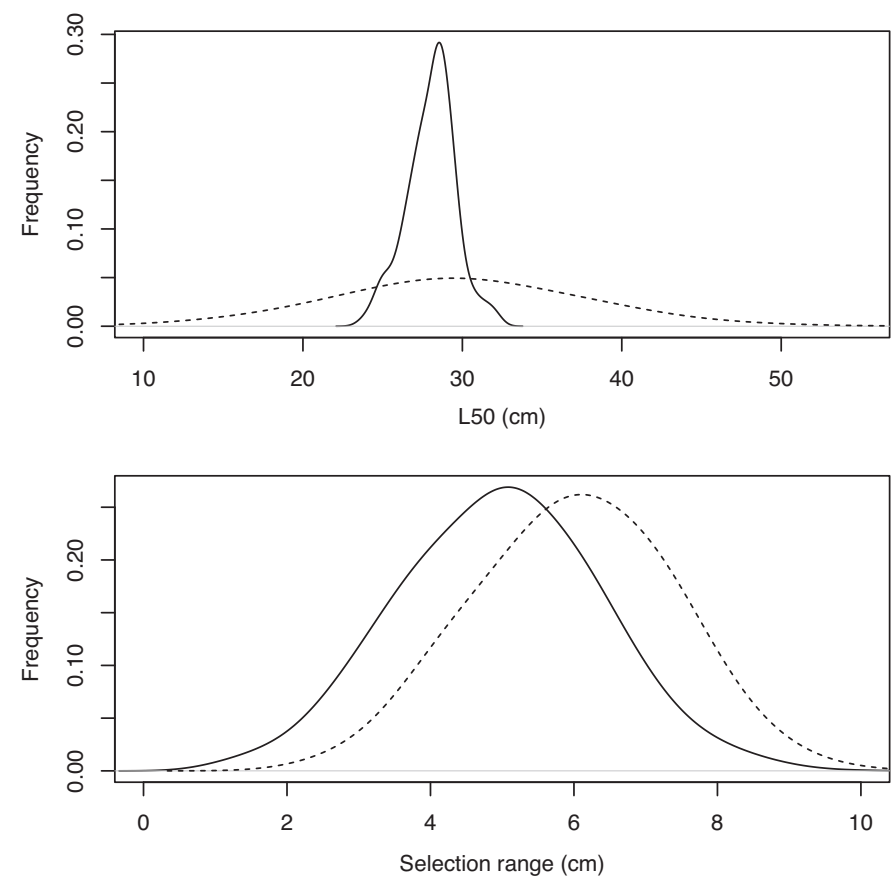

Fig. 6. Prior (dashed line) and posterior (solid line) distributions for $L_{50}$ and $s r^{*}$, the parameters of the fleet selectivity curve. 


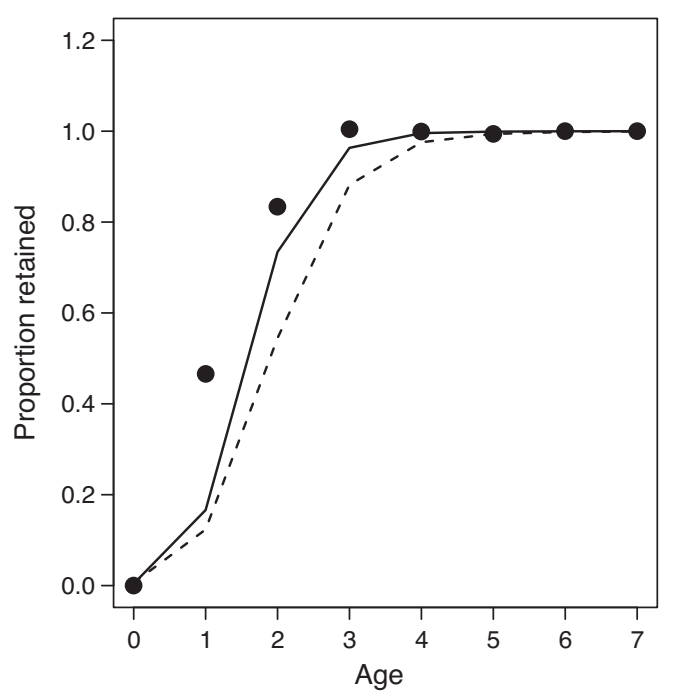

Fig. 7. Fleet selectivity based on the mean length at age over the reference period 1985-1994. The solid line shows the fitted (posterior mean) selection curve. The prior mean selectivity curve is indicated by the dashed line. The points show the selectivity estimated for the reference period from the ICES assessment.

\subsection{Catches and misreporting}

Overall the model estimates appear to capture the pattern of observed proportions of fish at each age (Fig. 8) providing some external corroboration of the model. It is noticeable that in recent years there is a marked increase in the proportion of fish aged four and older which is consistent with the much lower fishing mortality rates estimated for recent years (Fig. 1).

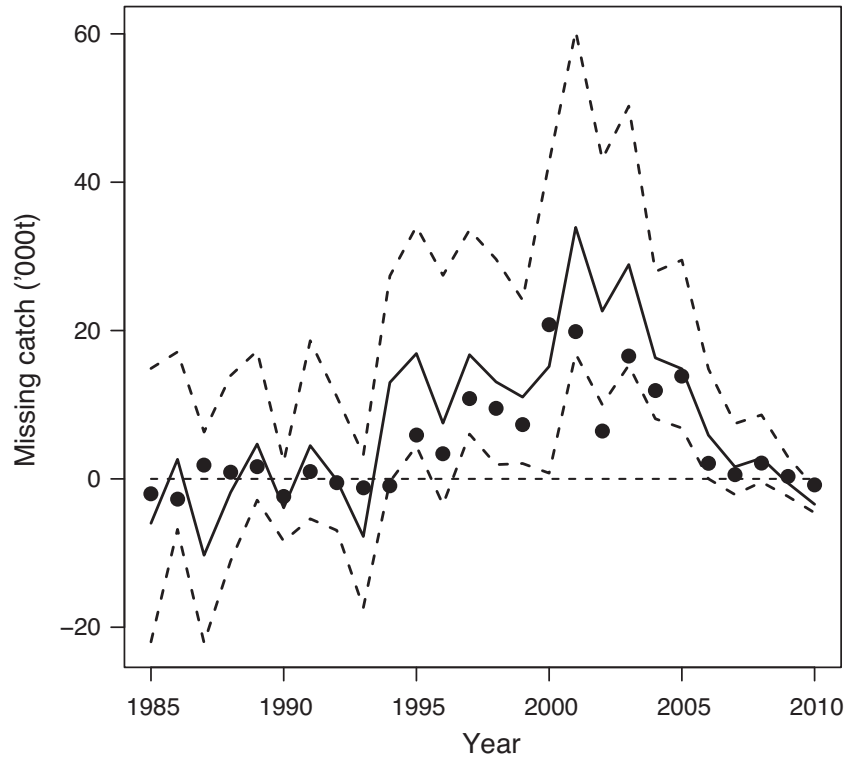

Fig. 9. Estimates of misreported or "missing" catch from the model (heavy line) compared to the ICES estimates (solid circles). Dashed lines show the $95 \%$ credible interval.

The estimates of missing catch peak at about 30,000 tonnes in 2001 but the credible interval is very large showing that there is high uncertainty associated with the values (Fig. 9). ICES estimates of missing catch are a little lower but fall within the $95 \% \mathrm{CI}$ and follow a very similar trend. To some degree the agreement between the model and ICES assessment is inevitable for the middle period because, like the model, the ICES assessment does not use catch

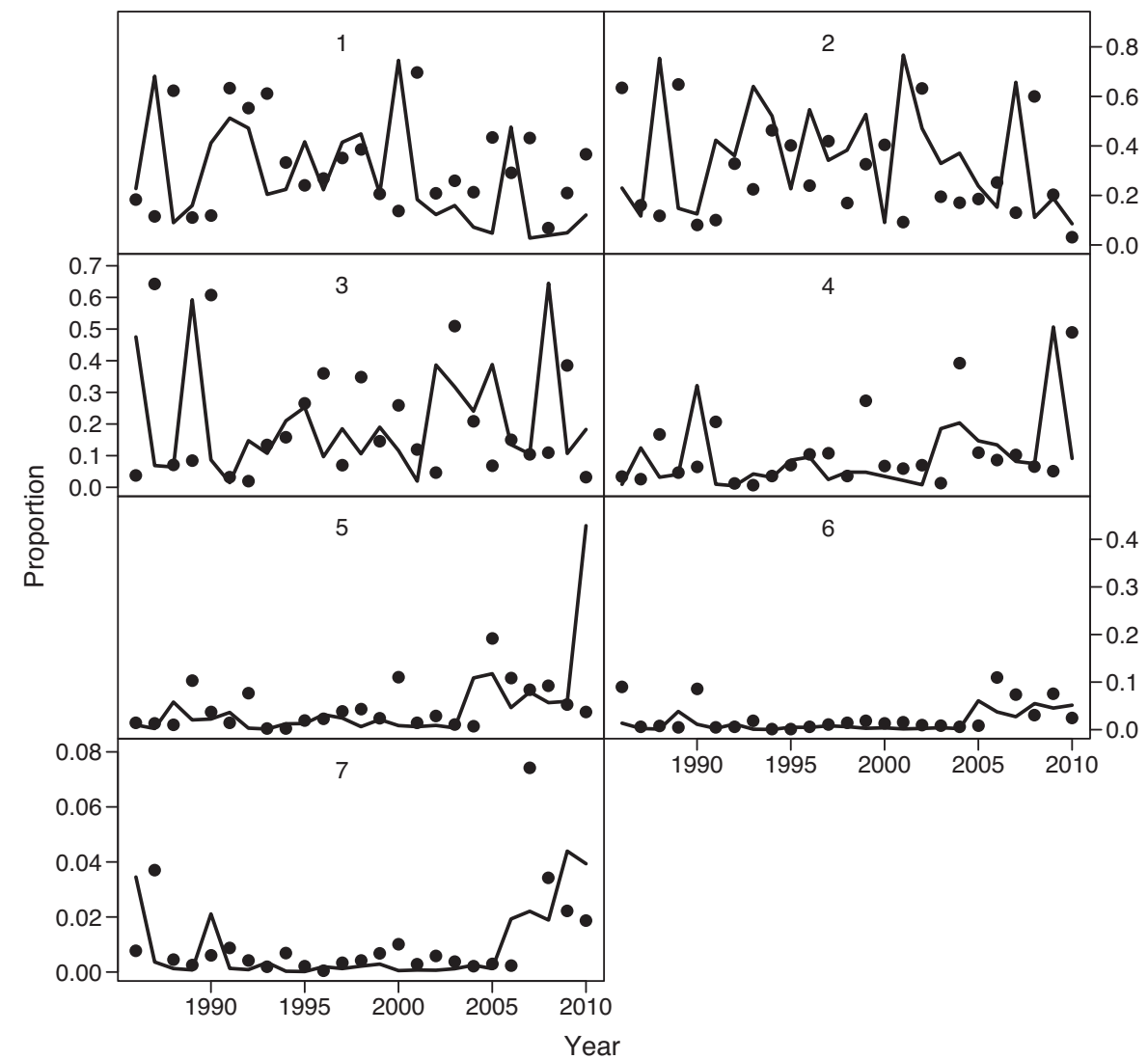

Fig. 8. Estimated proportion of fish at each age in the total catch (solid line) compared to the observed proportions (dots). Numbers in each panel indicate age classes. 


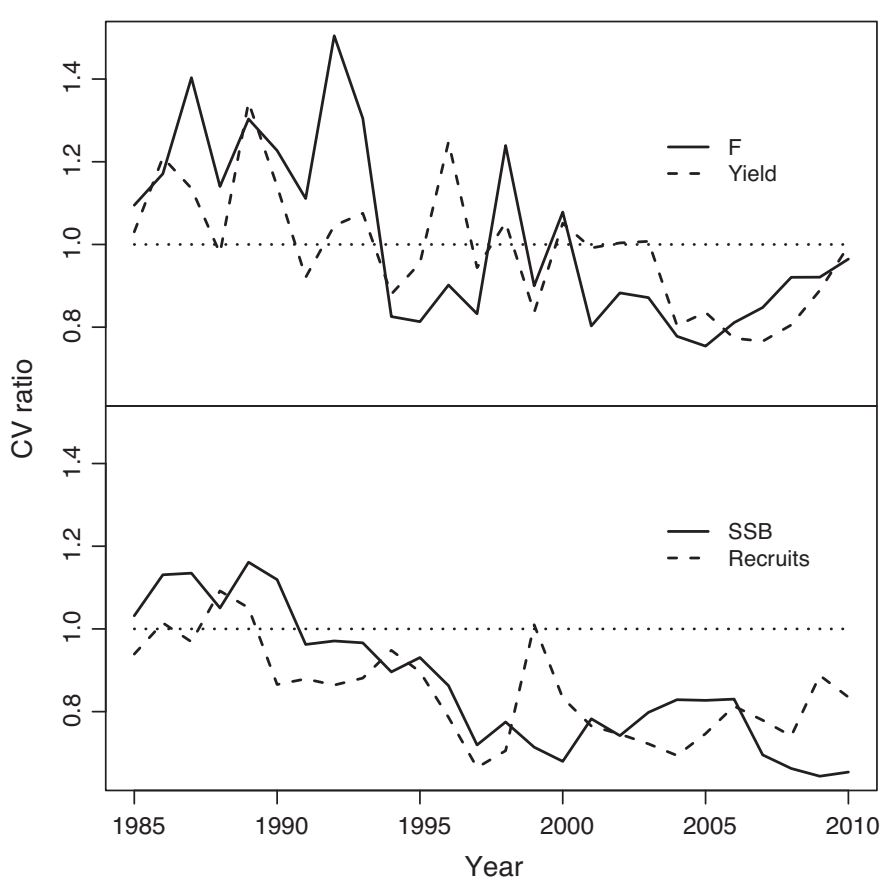

Fig. 10. Comparison of the model run with full data with a run using only the Sco1 survey. Upper panel shows the ratio for fishing mortality and total catch (yield). Lower panel shows the ratio for SSB and recruitment at age 1 .

at age data during the period of suspected high misreporting and relies on the two Scottish surveys for the estimate.

\subsection{Single versus multiple surveys}

The benefit of additional surveys can be judged by comparing model runs with single or multiple surveys. In the case of recruitment and SSB the inclusion of additional surveys clearly improves the $\mathrm{cv}$ from about the time the Sco2 survey index enters the analysis (Fig. 10). However, for fishing mortality and catch, there is very little improvement except for the period 2003-2008. This is perhaps not entirely surprising since $F$ and catch are derived values rather than direct estimates and are therefore subject to much greater uncertainty than recruitment and SSB.

\subsection{Natural mortality}

$M$ was modelled based on weight at age which produces higher values of $M$ at younger ages (Fig. 11) an effect which is expected if smaller fish are more vulnerable to predation mortality. The figure shows that for ages 2 and older, the posterior estimates for West of Scotland haddock are consistent with those from the adjacent North Sea derived from MSVPA and are somewhat larger than the conventional values of 0.2 . However, for the two youngest ages, there is a very large discrepancy between the two areas.

\section{Discussion}

The model developed here when applied to a stock subject to misreported catch is able to estimate quantities of interest such as fishing mortality rate and spawning stock biomass. It also appears that it is possible to obtain adequate estimates of fleet selectivity and hence estimate relative catch, which goes a long way to providing management information on the fishery. It would be a relatively simple matter to project the population forward to forecast short term population changes and catches under standard assumptions about fishing mortality and recruitment to provide

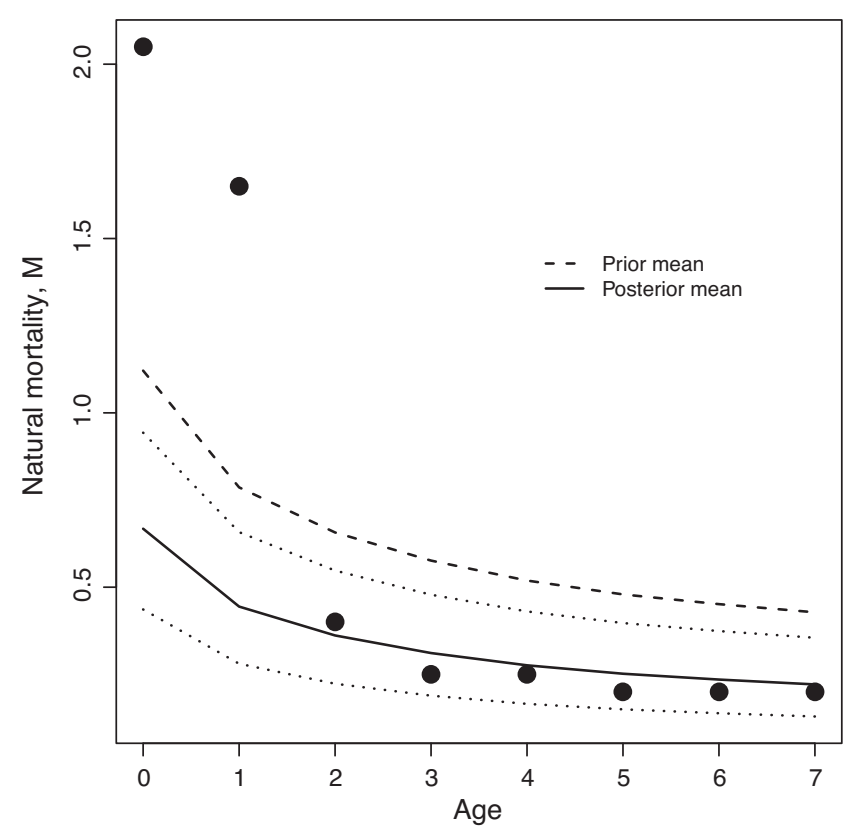

Fig. 11. Posterior values of $M$ based on mean weights at age for the reference period 1985-1994 shown as a heavy line with the $95 \%$ credible interval (dotted lines). The prior mean $M$ values are shown as a dashed line. Solid circles show the MSVPA values used in the ICES assessment for North Sea haddock (ICES, 2011b).

management advice. Reliance on commercial catch at age data in assessments can expose management of the stocks to deterioration in the data resulting from problems in sampling the catch or obtaining good estimates of quantities caught. This may be because substantial quantities of fish are discarded or because regulations provide an incentive to misreport. For example, cod (Gadus morhua) in the west of Scotland are subject to catch regulations so restrictive that most fish caught in recent years have been discarded (ICES, 2011b) making adequate sampling both for age and quantity problematic. In these cases methods based on surveys may be the next best option for the estimation of population parameters.

Where gross distortions in the catch data are suspected or observations are unavailable, a survey only approach is likely to be a useful tool. While this model does not attempt to estimate, explicitly, missing catch, it is possible to make such estimates (Fig. 9). They are however, highly uncertain and are probably better used to detect the presence or absence of misreporting rather than trying to estimate the absolute quantities. Both Patterson (1998) and Hammond and Trenkel (2005) suggest approaches to account for misreporting but these make fairly benign assumptions or knowledge about the scale of misreporting. The methods demonstrably can perform well under these assumptions and should be used where the conditions are satisfied.

The ability to estimate survey selectivity offers significant advantages over earlier methods such as Cook (1997) and SURBA (Needle, 2002) since it reduces the confounding effects of survey catchability, natural mortality and abundance. It is important when estimating fishing mortality and catch, that values for the selectivity of the surveys and fleets are realistic. It appears for this data set at least that the data do inform the estimates of the selectivities. This is perhaps somewhat surprising at first sight. However, where abundance indices increase with age (as may happen for the youngest fish) there is clear evidence that selectivity is lower at young ages. There is also substantial contrast in the data both in relation to recruitment and the change in fishing mortality which is likely to inform parameter estimation. Furthermore quite restrictive assumptions have been made about the shape of the selectivity curve which limits the flexibility in model. It is unlikely that if the 
selectivities at age were freely estimated, rather than being constrained to a logistic curve, that the model could estimate them. It is particularly important therefore that the assumption of logistic selectivity is adequate for the analysis. In the example used here, selectivity of this form is consistent with the ICES assessment where catch data have been used (Fig. 7).

The similarity of the posterior estimates of natural mortality with the corresponding values from the North Sea, which are estimated from real data, provides some corroboration of the model and is important in partitioning total mortality in order to estimate fishing mortality. A common assumption in other models is that it is a known constant. In the analysis here information is included in the form of a prior based on a meta-analysis. The prior mean lies above the $95 \%$ credible interval of the posterior distribution (Fig. 11) suggesting that the data do contain some information $M$. Lee et al. (2011) suggest that $M$ can be estimated from conventional stock assessment data based on simulated data sets and the apparent updating of the priors in this analysis does offer some support for this. However, it is not unusual for the results of model misspecification to emerge in estimates of $M$, so these values must be treated with caution. Millar and Meyer (2000), for example, using a similar Bayesian approach, assume that catches are known exactly and allow estimates of all other mortalities to be subsumed in $M$.

It is not uncommon to find that conflicting signals emerge from surveys and commercial catch even when they are well sampled (Schnute and Hilborn, 1993; Gavaris and Van Eeckhaute, 1998). Such conflicts may not be evident in comprehensive analyses where all the data are modelled simultaneously. Methods using survey data alone offer a way of estimating population parameters in the absence of commercial catch and may be used to detect differing signals in the data. They may have advantages particularly if catch data are severely biased or poorly sampled. However, they should not be regarded as a replacement for more comprehensive analyses, but an additional tool to explore available data.

Compared with a conventional assessment using catch at age data the model using survey data alone can produce a similar summary of stock status. It will perform best where, as in the example considered here, the signal to noise ratio in the survey data is high and where there is contrast in the fishing mortality rate. Survey data tend to have relatively large sampling errors compared to catch data because the sample size is much smaller so there will be a trade-off between the precision of the survey data and bias in the catch data in deciding when to use the model. Clearly high bias in the catch data and high precision in the survey will favour the method over models that rely on catch data. Having data from multiple surveys with different selectivities also helps in the estimation of commercial selectivity which is particularly important when trying to quantify the catch. These estimates, however, have low precision, at least in part because the information in the data on selectivity is limited, and only gross bias in the reported catches can be reliably detected.

\section{Acknowledgement}

This work was supported in part by MASTS small grant SG8.

\section{Appendix. Winbugs code used to fit the model}

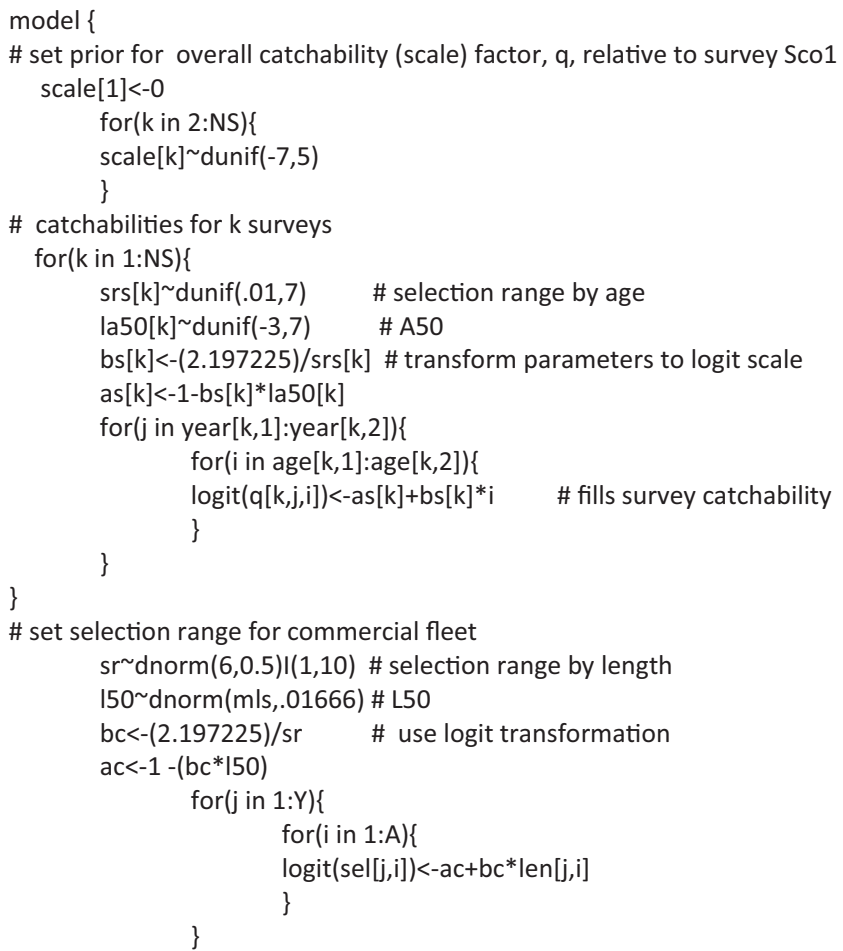

\#\#\#\#\#\#\#\#\#\#\#\#\#\#\#\#\#\#\#\#\#\#\#\#\#\#\#\#\# \# Lorenzen priors for natural mortality \# \#\#\#\#\#\#\#\#\#\#\#\#\#\#\#\#\#\#\#\#\#\#\#\#\#\#\#\# 


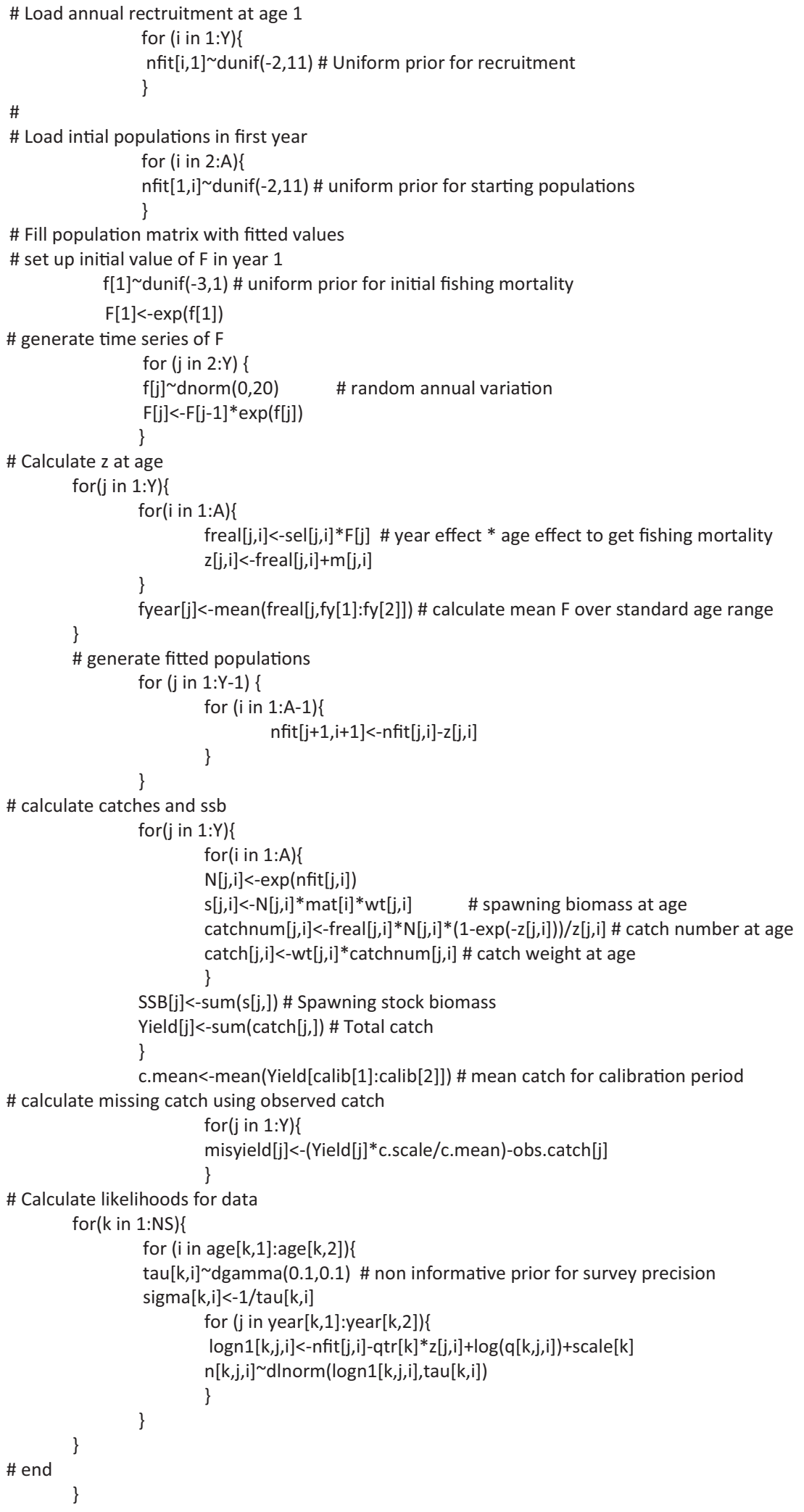

\section{References}

Andersen, K.P., Ursin, E., 1977. A multispecies extension to the Beverton and Holt theory of fishing, with accounts of phosphorous circulation and primary production. Meddelelser fra Danmarks Fiskeri-og Havundersogelser, N.S. 7, 319-435.

Beare, D.J., Needle, C.L., Burns, F., Reid, D.G., 2005. Using survey data independently from commercial data in stock assessment: an example using haddock in ICES Division VIa. ICES J. Mar. Sci. 62, 996-1005.

Cook, R.M., 1997. Stock trends in six North Sea stocks as revealed by an analysis of research vessel surveys. ICES J. Mar. Sci. 54, 924-933.

Coull, K.A., Jermyn, A.S., Newton, A.W., Henderson, G.I., Hall, W.B., 1989. Length/weight relationships for 88 species of fish encountered in the North East Atlantic. Scott. Fish. Res. Rep. 43.

Deriso, R.B., Quinn, T.J., Neal, P.R., 1985. Catch-age analysis with auxiliary information. Can. J. Fish. Aquat. Sci. 42, 815-824. 
Doubleday, W.G., 1981. A method for estimating the abundance of survivors of an exploited fish population using commercial catch at age and research vessel abundance indices. Can. Spec. Pub. Fish. Aquat. Sci. 58, 164-178.

FAO, 1994. A global assessment of fisheries by-catch and discards. Prepared by D.L. Alverson, M.H., Freeberg, S.A. Murawski,J.G. Pope. FAO Fisheries Technical Paper, no. 339, 233pp.

Fryer, R.J., 2001. TSA: is it the way? Working document for the Working Group on Methods of Fish Stock Assessment 2001, 15pp.

Gavaris, S., 1988. An adaptive framework for the estimation of population size. Can. Atl. Fish. Sci. Advisory Comm. (CAFSAC) Res. doc 88/29.

Gavaris, S., Van Eeckhaute, L., 1998. Diagnosing systematic errors in reported fishery catch. In Fishery stock assessment models. In: Funk, F., Quinn II, T.J., Heifetz, J., Ianelli, I., Powers, J.E., Schweigert, J.F., Sullivan, P.J., Zhang, C.-I. (Eds.). Alaska Sea Grant Program Report No. AK-SG-98-01, University of Alaska Fairbanks, 1998.

Graham, N., O’Neill, F.G., Fryer, R.J., Galbraith, R.D., Myklebust, A., 2004. Selectivity of a $120 \mathrm{~mm}$ diamond cod-end and the effect of inserting a rigid grid or a square mesh panel. Fish. Res. 67, 151L 161.

Gudmundsson, G., 2004. Time-series analysis of abundance indices of young fish. ICES J. Mar. Sci. 61, 176-183.

Gulland, J.A., 1965. Estimation of mortality rates. Annex to the Arctic Fisheries Working Group Report. ICES CM 1965 Doc. 3.

Hammond, T.R., Trenkel, V.M., 2005. Censored catch data in fisheries stock assessment. ICES J. Mar. Sci. 62, 1118-1130.

Hoenig, J.M., 1983. Empirical use of longevity data to estimate total mortality rates. Fish. Bull. 82, 898-903.

ICES, 2010. Report of the ICES Advisory Committee 2010. Book 5.

ICES, 2011a. Report of the Working Group for Celtic Seas Ecoregion (WGCSE). ICES, CM 2011/ACOM:12.

ICES, 2011b. Report of the Working Group on the Assessment of Demersal Stocks in the North Sea and Skagerrak (WGNSSK). ICES CM 2011/ACOM:13.

Lee, H.-H., Maunder, M.N., Piner, K.R., Methot, R.D., 2011. Estimating natural mortality within a fisheries stock assessment model: an evaluation using simulation analysis based on twelve stock assessments. Fish. Res. 109, 89-94.

Lorenzen, K., 1996. The relationship between body weight and natural mortality in juvenile and adult fish: a comparison of natural ecosystems and aquaculture. J. Fish. Biol. 49, 627-647.
Lorenzen, K., 2000. Allometry of natural mortality as a basis for assessing optimal release size in fish-stocking programmes. Can. J. Fish. Aquat. Sci. 57, 2374-2381.

Lunn, D.J., Thomas, A., Best, N., Spiegelhalter, D., 2000. WinBUGS - a Bayesian modelling framework: concepts, structure, and extensibility. Stat. Comput. 10 325-337.

Methot, R.D., 1990. Synthesis model: an adaptive framework for analysis of diverse stock assessment data. Int. North. Pac. Fish. Comm. Bull. 50, 259-277.

Millar, R.B., Meyer, R., 2000. Bayesian state-space modeling of age-structured data: fitting a model is just the beginning. Can. J. Fish. Aquat. Sci 57, 43-50.

Myers, R.A., Doyle, R.W., 1983. Predicting natural mortality rates and reproductionmortality trade-offs from fish life history data. Can. J. Fish. Aquat. Sci. 40 612-629.

Needle C.L., 2002. Working Document WD2 to the ICES Working Group on the Assessment of Demersal Stocks in the North Sea and Skagerrak. Copenhagen, ICES. Preliminary analyses of survey indices for whiting in VI and VIId.

Özbilgin, H., Ferro, R.S.T., Robertson, J.H.B., Holtrop, G., Kynoch, R.J., 2006. Seasonal variation in trawl codend selection of northern North Sea haddock. ICES J. Mar. Sci. 63, 737-748.

Patterson, K., 1998. Assessing fish stocks when catches are misreported: model, simulation tests, and application to cod haddock and whiting in the ICES area. ICES J. Mar. Sci. 55, 78-891.

Pauly, D., 1980. On the interrelationships between natural mortality, growth parameters, and mean environmental temperature for 175 fish stocks. J. Cons. Int. Explor. Mer. 3, 175-192.

Pope, J.G., 1979. A modified cohort analysis in which constant mortality is replaced by estimates of predation levels. ICES Doc. CM 1979/H: 16, 8pp.

Pope, J.G., Shepherd, J.G., 1982. A simple method for the consistent interpretation of catch at age data. J. Cons. Int. Explor. Mer. 42, 129-151.

Schnute, Jon, T., Hilborn, R., 1993. Analysis of contradictory data sources in fish stock assessment. Can. J. Fish. Aquat. Sci. 50, 1916-1923.

Shepherd, J.G., 1999. Extended survivors analysis: an improved method for the analysis of catch-at-age data and abundance indices. ICES J. Mar. Sci. 56, 584-591.

Stratoudakis, Y., Fryer, R.J., Cook, R.M., Pierce, G.J., 1999. Fish discarded from Scottish demersal vessels: estimators of total discards and annual estimates for targeted gadoids. ICES J. Mar. Sci. 56, 592-605.

Vetter, E.F., 1988. Estimation of natural mortality in fish stocks: a review. Fish. Bull. $86,25-43$ 